\title{
Exercise testing of pre-school children using the Bruce treadmill protocol: new reference values
}

\author{
Monique H. M. van der Cammen-van Zijp • Hanneke IJsselstijn • Tim Takken • \\ Sten P. Willemsen · Dick Tibboel · Henk J. Stam • Rita J. G. van den Berg-Emons
}

Accepted: 28 September 2009 / Published online: 11 October 2009

(C) The Author(s) 2009. This article is published with open access at Springerlink.com

\begin{abstract}
The Bruce treadmill protocol is an often-used exercise test for children and adults. Few and mainly old normative data are available for young children. In this cross-sectional observational study we determined new reference values for the original Bruce protocol in children aged 4 and 5 years. Furthermore, we compared the original protocol with the so-called 'half Bruce' protocol. In the Netherlands this half-Bruce protocol is often used for young children because of the rather large increments in workload in the original protocol. Seventy-eight healthy
\end{abstract}

Communicated by Susan Ward.

M. H. M. van der Cammen-van Zijp ( $\varangle)$

Department of Physical Therapy,

Subdivision Pediatric Physical Therapy,

Room Sk 0327, Sophia Children's Hospital,

Erasmus MC, University Medical Center Rotterdam,

Dr. Molewaterplein 60, 3015 GJ Rotterdam, The Netherlands

e-mail: m.vandercammen@erasmusmc.nl

M. H. M. van der Cammen-van Zijp · H. IJsselstijn · D. Tibboel Department of Pediatric Surgery, Sophia Children's Hospital, Erasmus MC, University Medical Center Rotterdam,

Rotterdam, The Netherlands

T. Takken

Department of Pediatric Physical Therapy and Exercise Physiology, Wilhelmina Children's Hospital,

University Medical Center Utrecht, Utrecht, The Netherlands

S. P. Willemsen

Department of Biostatistics, Erasmus MC, University Medical Center Rotterdam,

Rotterdam, The Netherlands

H. J. Stam · R. J. G. van den Berg-Emons Department of Rehabilitation Medicine,

Erasmus MC, University Medical Center Rotterdam,

Rotterdam, The Netherlands
Dutch children participated. The maximal endurance time was the criterion of exercise capacity. The new reference values for the original Bruce protocol are presented as reference centiles. The mean (SD) endurance time using the original protocol was 10.2 (SD 1.5) min.; this was 9.4 (1.3) $\min$. for the half-Bruce protocol. The mean difference was 50 seconds (95\% CI: $29-71 \mathrm{~s}, P<0.001)$. So, for children aged 4 and 5 years the endurance times obtained with the original and half-Bruce protocol are different and should not be considered interchangeable. Our new reference values can be used as reference values for the original Bruce protocol.

Keywords Exercise test - Physical endurance ·

Pre-school child

\section{Introduction}

Exercise testing provides information on exercise capacity and facilitates assessment of pathophysiologic characteristics, effectiveness of medication, and risk of potential disease (Bar-Or 1983). Since children have relatively undeveloped knee extensors, treadmill testing is preferred over cycle ergometry in young children (Bar-Or 1983; Bar-Or and Rowland 2004). An often used protocol is the Bruce treadmill protocol, which was originally designed for adults (Bruce et al. 1973) but is now also applied worldwide for children from the age of 4 years (Wessel et al. 2001).

Reference values for children aged 4 and 5 years have been established mainly in small study samples (Binkhorst et al. 1992; Chatrath et al. 2002; Wessel et al. 2001). Only Cumming et al. (1978) presented values obtained in a larger group (up to forty $4-5$ years olds). However, these data date back more than 30 years and might therefore not be appropriate for contemporary children (Wessel et al. 2001). 
Recently, we showed that the maximal exercise capacity in children from 6 to 10 years seems to have deteriorated during the past 20 years. In these children, body mass index (BMI) was negatively, and intense sports participation was positively associated with endurance time (van der Cammen-van Zijp et al. 2009).

The original Bruce treadmill protocol has rather large increments in workload between stages. It is therefore that in the Netherlands many pediatric physical therapists use the so-called 'half Bruce' treadmill protocol for young children. This modified protocol has $1.5 \mathrm{~min}$ stages instead of 3 min. stages and smaller speed increments (Table 1). As reference values for the modified protocol are lacking, those for the original Bruce protocol are then applied (Takken 2007). We hypothesize, however, that because of the difference in workload, children's maximal endurance times using this half-Bruce treadmill protocol are lower than those for the original Bruce protocol.

In view of these observations, the primary objective of the present study was to determine reference values for healthy Dutch children aged between 4 and 5 years using the original Bruce treadmill protocol. The secondary objectives were: (1) to compare the endurance times on the original and half-Bruce protocol; (2) to evaluate BMI, height for age, socio-economic status (SES), ethnicity, sports participation, and school transport habits as possible determinants of exercise capacity.

\section{Subjects and methods}

We recruited 80 healthy children aged $4-5$ years (39 boys and 41 girls) from three different primary schools in the

Table 1 Test details for the Bruce and the half-Bruce treadmill protocol

\begin{tabular}{|c|c|c|c|c|c|c|c|}
\hline \multicolumn{4}{|l|}{ Bruce } & \multicolumn{4}{|c|}{ Half Bruce } \\
\hline Stage & $\begin{array}{l}\text { Speed } \\
(\mathrm{km} / \mathrm{h})\end{array}$ & $\begin{array}{l}\text { Grade } \\
(\%)\end{array}$ & $\begin{array}{l}\text { Time } \\
(\min )\end{array}$ & Stage & $\begin{array}{l}\text { Speed } \\
(\mathrm{km} / \mathrm{h})\end{array}$ & $\begin{array}{l}\text { Grade } \\
(\%)\end{array}$ & $\begin{array}{l}\text { Time } \\
\text { (min) }\end{array}$ \\
\hline \multirow[t]{2}{*}{ I } & 2.7 & 10 & 3 & I & 2.7 & 10 & 1.5 \\
\hline & & & & II & 3.4 & 11 & 1.5 \\
\hline \multirow[t]{2}{*}{ II } & 4 & 12 & 3 & III & 4 & 12 & 1.5 \\
\hline & & & & IV & 4.7 & 13 & 1.5 \\
\hline \multirow[t]{2}{*}{ III } & 5.4 & 14 & 3 & V & 5.4 & 14 & 1.5 \\
\hline & & & & VI & 6 & 15 & 1.5 \\
\hline \multirow[t]{2}{*}{ IV } & 6.7 & 16 & 3 & VII & 6.7 & 16 & 1.5 \\
\hline & & & & VIII & 7.3 & 17 & 1.5 \\
\hline \multirow[t]{2}{*}{ V } & 8 & 18 & 3 & IX & 8 & 18 & 1.5 \\
\hline & & & & $X$ & 8.4 & 19 & 1.5 \\
\hline \multirow[t]{2}{*}{ VI } & 8.8 & 20 & 3 & XI & 8.8 & 20 & 1.5 \\
\hline & & & & XII & 9.2 & 21 & 1.5 \\
\hline
\end{tabular}

Southwestern part of the Netherlands. The schools were located in both urban and suburban regions. Children were considered to be healthy when parents declared that their child did not suffer from pulmonary or cardiovascular disease or a health condition treated by a medical specialist. We excluded children with impaired motor function; children who used medication affecting exercise capacity; and obese children [BMI above +2 standard deviation scores (SDS) compared to Dutch norms (Fredriks et al. 2000b)]. Also excluded from analysis were children who did not fulfill the criteria of maximal exercise performance as described below. As two children were excluded, one for cardiovascular disease and one due to sub maximal performance, 78 children were evaluated.

\section{Study design}

This cross-sectional observational study was performed between July 2006 and March 2008. We used two different study designs: (1) To compare the original Bruce protocol with the half-Bruce protocol 53 children were randomly allocated to start with one of the two exercise test protocols (Fig. 1; Group I). They performed both test protocols within a period of 1-14 days. (2) To obtain reference values for the original Bruce protocol, we studied 53 children who performed the Bruce treadmill protocol without prior experience on exercise testing (Fig. 1; Group II).

Most children were tested in a quiet room at their own schools. Some, living close by, performed the test in the hospital's exercise room. In accordance with daily practice and the procedure applied in former research, they were not given the opportunity to practise.

Parents of all participants filled out a pre-test questionnaire as established previously (Van der Cammen-van Zijp et al. 2009). Parental estimation of their child's fitness level

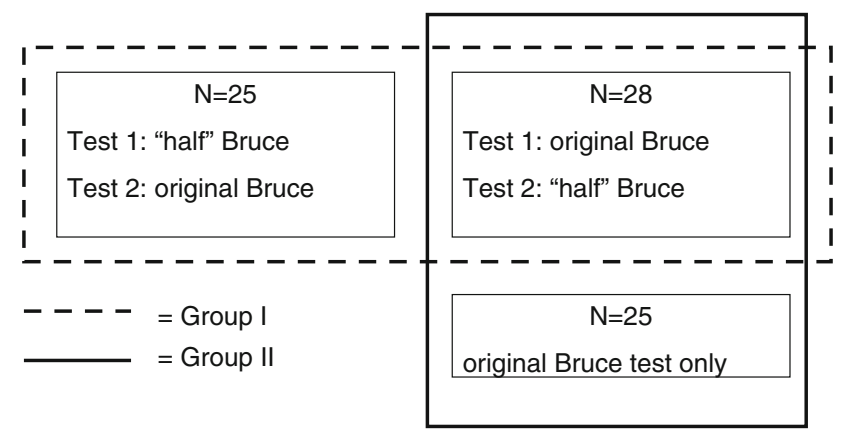

Fig. 1 Number of participants studied for each protocol. The square with dashed lines indicates the 53 subjects, who were tested twice in order to evaluate the difference between the original and the "halfBruce' (Group I). The square with solid lines indicates the 53 subjects, who were included in the determination of reference values (Group II): those who were tested twice and started with the original protocol $(n=28)$ and those who were only tested once $(n=25)$ 
was classified as higher than, equal to or less than that for children of the same age.

The Erasmus MC Medical Ethical Review Board approved the study. Written informed consent was obtained from all parents or guardians.

The treadmill tests

The children performed the maximal exercise tests on a motor-driven treadmill (En Mill, Enraf Nonius BV, Rotterdam, The Netherlands), programed for increases in angle of inclination and speed for the original Bruce protocol or for the half-Bruce protocol, as applicable (Table 1).

A physical therapist trained to apply Basic Life Support to children supervised all tests. In view of the children's young age, we slightly modified the procedure by permitting them to hold the guardrail to maintain body position near the center of the moving belt. From experience we knew that otherwise they would have balance problems. We encouraged the children to perform to voluntary exhaustion. Thereafter, the children continued walking at a slope of $0 \%$ and a speed of $2 \mathrm{~km} / \mathrm{h}$ for $2 \mathrm{~min}$. The maximal endurance time (in minutes, two decimals) served as criterion of exercise capacity. Before and during a test, heart rate (HR) was monitored with a pulse oximeter (MARS, motion artifact system; type 2001, Respironics Novametrix, Murrysville, PA) attached to the index finger. HR of $\geq 185$ beats per minute (bpm) at the end of the test (Karila et al. 2001) or loss of walking-coordination was considered to indicate maximal performance. If any technical problems precluded measurements at the end of the test, we considered maximal performance to be based on HR as monitored in the pre-final stage of the test.

Determinants of exercise capacity

\section{Pre-test questionnaire}

The parental pre-test questionnaire included questions on school transport habits, one-way travel distance, sports participation, passive smoking, ethnic origin, and SES. For children who cycled to school 5 days per week a weekly commuting distance (in $\mathrm{km}$ ) was calculated by multiplying the one-way travel distance by 10 . Sports participation was classified into low (only school gymnastic lessons), moderate (gymnastic lessons and participation in organized sports up to $2 \mathrm{~h}$ weekly), or high (gymnastic lessons and more than $2 \mathrm{~h}$ of organized sports weekly). We classified ethnic origin using the definition of Statistics Netherlands into 'Dutch', 'Western background' or 'non-Western background' (Statistics Netherlands 2004). SES was classified into: 'low', 'middle', and 'high' (Statistics Netherlands 2001).

\section{Pre-test evaluation}

A pre-test evaluation included measurement of height $(\mathrm{cm})$ and body mass (kg) using a stadiometer (Seca 206, Seca, Hamburg, Germany) and a scale (Beurer PS-16, Beurer, Ulm, Germany), respectively. The Dutch Growth Analyser, version 3.0 (Dutch Growth Foundation, Rotterdam) served to calculate SDS for height and BMI, on the basis of Dutch reference values published in 2000 (Fredriks et al. 2000a).

\section{Data analysis}

Data for children in Group II served to establish reference values for the original Bruce protocol. Age-related reference centiles were constructed according to Altman (Altman 1993). In brief, the endurance time is assumed to follow a normal distribution for a given age and sex. First, the mean endurance time is modeled as a function of age. The absolute residuals of this regression are then regressed on age to provide an estimate of the standard deviation of the endurance time as a function of age. Means and standard deviations were combined to give estimates for the centiles (centile $=$ mean $+Z \times \mathrm{SD}$, where $Z$ is the corresponding centile of the standard normal distribution).

One-sample $t$ tests were performed to analyze differences in anthropometric parameters between the norm group (Group II) and the general population norms (Fredriks et al. 2000a).

For participants in Group I we compared the effects of the two different Bruce protocols using ANOVA for repeated measurements (Senn 2002). We tested whether there was a learning effect; i.e., whether a subject after a first Bruce test-irrespective of what protocol-would score better on the second test than he or she would have done when performing this test without prior experience. Finally, we tested to see if there was a sequence by treatment interaction; i.e., whether the size of the learning effect depended on which form of the Bruce test was taken first. Group comparisons (differences in the subject characteristics between subgroups in Group I; Fig. 1), were performed with the independent $t$ test, Mann-Whitney $U$ test or Chisquare test where appropriate.

To evaluate determinants of exercise capacity corrected for sex and age we first calculated the SDS of the endurance time, i.e., the difference between the observed and predicted value divided by the standard deviation from the reference values. Then, we investigated whether differences in endurance time in the children of group II, could be explained by SDS height, SDS BMI, SES, ethnicity, sports participation or school transport habits. We used linear regression analyses with the SDS of the maximal endurance time as outcome variable. 
Paired-samples $t$ tests were used to evaluate the differences in HR during the original and half-Bruce protocol. $P$ values $<0.01$ were considered statistically significant. Data presented are mean (SD), unless stated otherwise. Statistical analyses were performed using SPSS 15.0 for Windows.

\section{Results}

Reference values for exercise capacity

The characteristics of the children from the reference group (Group II) are shown in Table 2. None of the children were exposed to smoking. SDS height and BMI did not significantly differ from general population norms.

The percentile scores for endurance time are presented in Table 3. The age-related reference centiles are graphically shown in Fig. 2a, b. A good fit could be achieved using a linear function for the mean. Variance did not

Table 2 Characteristics of participants for establishment of reference data (group II)

\begin{tabular}{|c|c|c|c|}
\hline & Boys & Girls & Total \\
\hline$n$ & 25 & 28 & 53 \\
\hline Age in years, mean $(\mathrm{SD})$ & $5.0(0.6)$ & $5.0(0.6)$ & $5.0(0.6)$ \\
\hline Height SD-scores, mean (SD) & $-0.26(1.0)$ & $0.42(0.9)$ & $0.09(1.0)$ \\
\hline BMI SD-scores, mean (SD) & $0.24(0.8)$ & $0.16(1.0)$ & $0.20(0.9)$ \\
\hline \multicolumn{4}{|c|}{ Socio-economic status (SES), $n(\%)$} \\
\hline High & $17(68)$ & $22(78.6)$ & $39(73.6)$ \\
\hline Middle & $8(32)$ & $5(17.8)$ & $13(24.5)$ \\
\hline Low & - & $1(3.6)$ & $1(1.9)$ \\
\hline \multicolumn{4}{|l|}{ Ethnic group, $n(\%)$} \\
\hline Dutch & $20(80)$ & $25(89.3)$ & 45 (84.9) \\
\hline Western background & $1(4)$ & $1(3.6)$ & $2(3.8)$ \\
\hline Non Western background & $4(16)$ & $2(7.1)$ & $6(11.3)$ \\
\hline \multicolumn{4}{|l|}{ Sports participation, $n(\%)$} \\
\hline High & $1(4)$ & $1(3.6)$ & $2(3.8)$ \\
\hline Moderate & $18(72)$ & $19(67.9)$ & $37(69.8)$ \\
\hline Low & $6(24)$ & $8(28.6)$ & $14(26.4)$ \\
\hline \multicolumn{4}{|l|}{ Home/school commuting, $n(\%)$} \\
\hline $\begin{array}{l}\text { By car or sitting at } \\
\text { parent's bicycle }\end{array}$ & $6(24)$ & $9(32.1)$ & $15(28.3)$ \\
\hline Walking or public transport & $13(52)$ & $7(25)$ & $20(37.7)$ \\
\hline Cycling themselves & $6(24)$ & $11(39.3)$ & $17(32.1)$ \\
\hline Missing & - & $1(3.6)$ & $1(1.9)$ \\
\hline \multicolumn{4}{|c|}{ Parental estimation of fitness level, $n(\%)$} \\
\hline Better than peers & $4(16)$ & $1(3.6)$ & $5(9.4)$ \\
\hline Similar than peers & $19(76)$ & $26(92.8)$ & $45(84.9)$ \\
\hline Worse than peers & $1(4.0)$ & $1(3.6)$ & $2(3.8)$ \\
\hline Missing & $1(4.0)$ & - & $1(1.9)$ \\
\hline
\end{tabular}

depend on age, so we could use a constant variance. At the end of the test HR was reliably recorded in 36 children (70\%). It was a mean of $191 \mathrm{bpm}$ (SD 11) in boys and a mean of $195 \mathrm{bpm}$ (SD 9) in girls, indicating maximal performance Technical problems precluded reliable recording of $\mathrm{HR}$ in the final stage of the test for the other 17 children, but, based on HR in their pre-final stage or loss of coordination, we considered performance in those children also as maximal.

Difference between the two protocols

Children in subgroups of Group I (Fig. 1) did not differ with respect to age, anthropometric data, exposure to smoking, SES, ethnicity, sports participation, school transport habits, and parental estimation of fitness level (data not shown). The mean endurance time using the original Bruce protocol was $10.2 \mathrm{~min}$ (SD 1.5); that was $9.4 \mathrm{~min}$ (SD 1.3) for the half-Bruce protocol. The mean difference was $50 \mathrm{~s}(95 \%$ CI: $29-71 \mathrm{~s} ; P<0.001)$. We noted a significant learning effect of 47 s $(95 \%$ CI: $26-68 \mathrm{~s} ; P<0.001)$. There was no significant interaction between sequence and the results of the Bruce tests $(P=0.851)$.

The HR was monitored before and during both exercise tests. In both protocols the children's mean HR at baseline was 99 (SD 13) bpm. At maximal performance the mean HR in both protocols was 193 (SD 9) bpm. Figure 3 shows the HR increase patterns in the two different protocols.

Determinants of exercise capacity

Linear regression analysis showed no significant relations between endurance SDS and SDS height $\left(R^{2}=0.002\right.$, $P=0.764), \quad$ SDS BMI $\left(R^{2}=0.001, \quad P=0.812\right), \quad$ SES $\left(R^{2}=0.013, P=0.722\right)$, ethnicity $\left(R^{2}=0.020, P=0.616\right)$, sports participation $\left(R^{2}=0.081, P=0.127\right)$ or school transport habits $\left(R^{2}=0.044, P=0.336\right)$ for the 53 children in group II.

\section{Discussion}

The present cross-sectional observational study provides an update of reference values for the original Bruce treadmill protocol in healthy children aged 4 and 5 years. An update was felt necessary as only few and mainly old normative values were available for these young children. Endurance times on the half-Bruce protocol were lower than that on the original Bruce protocol. SDS height, SDS BMI, SES, ethnicity, sports participation and school transport habits were not significantly associated with the SDS endurance time. 
Table 3 Endurance times for boys and girls
Endurance times in minutes, two decimals. SD boys: $1.64 \mathrm{~min}$; SD girls: $1.20 \mathrm{~min}$

\begin{tabular}{lllllllll}
\hline Gender & Age (years) & p 5 & p 10 & p 25 & p 50 & p 75 & p 90 & p 95 \\
\hline Boys & 4.00 & 5.55 & 6.14 & 7.14 & 8.25 & 9.36 & 10.36 & 10.96 \\
& 4.25 & 5.94 & 6.54 & 7.54 & 8.65 & 9.76 & 10.76 & 11.35 \\
& 4.50 & 6.34 & 6.94 & 7.94 & 9.05 & 10.16 & 11.15 & 11.75 \\
& 4.75 & 6.74 & 7.34 & 8.33 & 9.44 & 10.55 & 11.55 & 12.15 \\
& 5.00 & 7.14 & 7.73 & 8.73 & 9.84 & 10.95 & 11.95 & 12.55 \\
& 5.25 & 7.53 & 8.13 & 9.13 & 10.24 & 11.35 & 12.35 & 12.94 \\
& 5.50 & 7.93 & 8.53 & 9.53 & 10.64 & 11.74 & 12.74 & 13.34 \\
Girls & 5.75 & 8.33 & 8.92 & 9.92 & 11.03 & 12.14 & 13.14 & 13.74 \\
& 4.00 & 7.76 & 8.20 & 8.92 & 9.73 & 10.54 & 11.27 & 11.70 \\
& 4.25 & 7.86 & 8.29 & 9.02 & 9.83 & 10.64 & 11.36 & 11.80 \\
& 4.50 & 7.95 & 8.39 & 9.12 & 9.92 & 10.73 & 11.46 & 11.89 \\
& 4.75 & 8.05 & 8.48 & 9.21 & 10.02 & 10.83 & 11.55 & 11.99 \\
& 5.00 & 8.14 & 8.58 & 9.31 & 10.12 & 10.92 & 11.65 & 12.09 \\
& 5.25 & 8.24 & 8.68 & 9.40 & 10.21 & 11.02 & 11.75 & 12.18 \\
& 5.50 & 8.34 & 8.77 & 9.50 & 10.31 & 11.11 & 11.84 & 12.28 \\
& 5.75 & 8.43 & 8.87 & 9.59 & 10.40 & 11.21 & 11.94 & 12.37 \\
\hline
\end{tabular}

We slightly modified the test procedure by permitting the children to hold the guardrails. In our experience, walking on the treadmill with increments of speed and inclination till maximal performance without rail holding is too difficult for many children aged 4 or 5 years. This is why we, in accordance with daily practice, preferred the safety of holding the rail in these young children. Our strategy enabled more young children to perform this maximal exercise test. Rail holding, however, is known to increase endurance time and reduce physiological strain (e.g. HR, $\mathrm{VO}_{2}$ ) during sub maximal exercise (Ellestad 2003). And recently, we showed that 6-year-old children, who were not able to perform the Bruce protocol without holding the guardrail, reached longer endurance times than did their age peers (Van der Cammen-van Zijp et al. 2009). A limitation of our modification is that results cannot easily be compared with those from other studies, because rail holding is not always permitted. Thus, and also in view of other differences in study conditions and the small groups in some studies (Binkhorst et al.1992, Chatrath et al. 2002) statistical analysis of the differences between studies is not possible. However, deterioration in maximal exercise capacity in Dutch children seems to be likely: for boys the mean endurance time was $9.6 \mathrm{~min}$ in the present study and $11.2 \mathrm{~min}$ in 1992. For girls the difference was less: $10.1 \mathrm{~min}$ in the present study and $10.6 \mathrm{~min}$ in 1992 (Binkhorst et al. 1992). The difference would have been even larger when we had not allowed our children to hold the guardrail. Two studies in North-American children report on exercise testing using the Bruce protocol as well (Cumming et al. 1978; Chatrath et al. 2002). Children in 2002 showed a shorter endurance time on the Bruce treadmill test than those tested in 1977.
These findings are consonant with our recent findings that maximal exercise capacity seems to have deteriorated during the past 20 years in children from 6 to 10 years (van der Cammen-van Zijp et al. 2009). Longitudinal follow-up studies should be performed to confirm the cross-sectional data of the current study.

In the present study we were not able to identify any determinants of maximal exercise capacity. For children aged 6-13 years, however, we earlier found a positive interaction between intensive sports participation and maximal exercise capacity (van der Cammen-van Zijp et al. 2009). In children aged 4 and 5 years, the range in sports participation is rather small, which may explain why we did not find a correlation between sports participation and exercise capacity.

One of the secondary aims of the study was to compare the endurance times in the two protocols applied. We found a significant difference of $50 \mathrm{~s}$ in endurance time in favor of the original Bruce protocol, which can be explained by the difference in increments in speed and grade between the protocols. In the original protocol, the concurrent speed and grade at the end of each stage is lower than that in the half-Bruce protocol (see Table 1). This probably explains the observed faster increase in HR in the half-Bruce protocol (Fig. 3). This phenomenon confirms that there is a difference in strain between the two exercise protocols, resulting in a lower endurance time in the half protocol. On that basis, seeing that these 4- and 5-year-olds could perform the original protocol properly, we think there is no need to use the half-Bruce protocol when using the maximal endurance time as outcome parameter. 

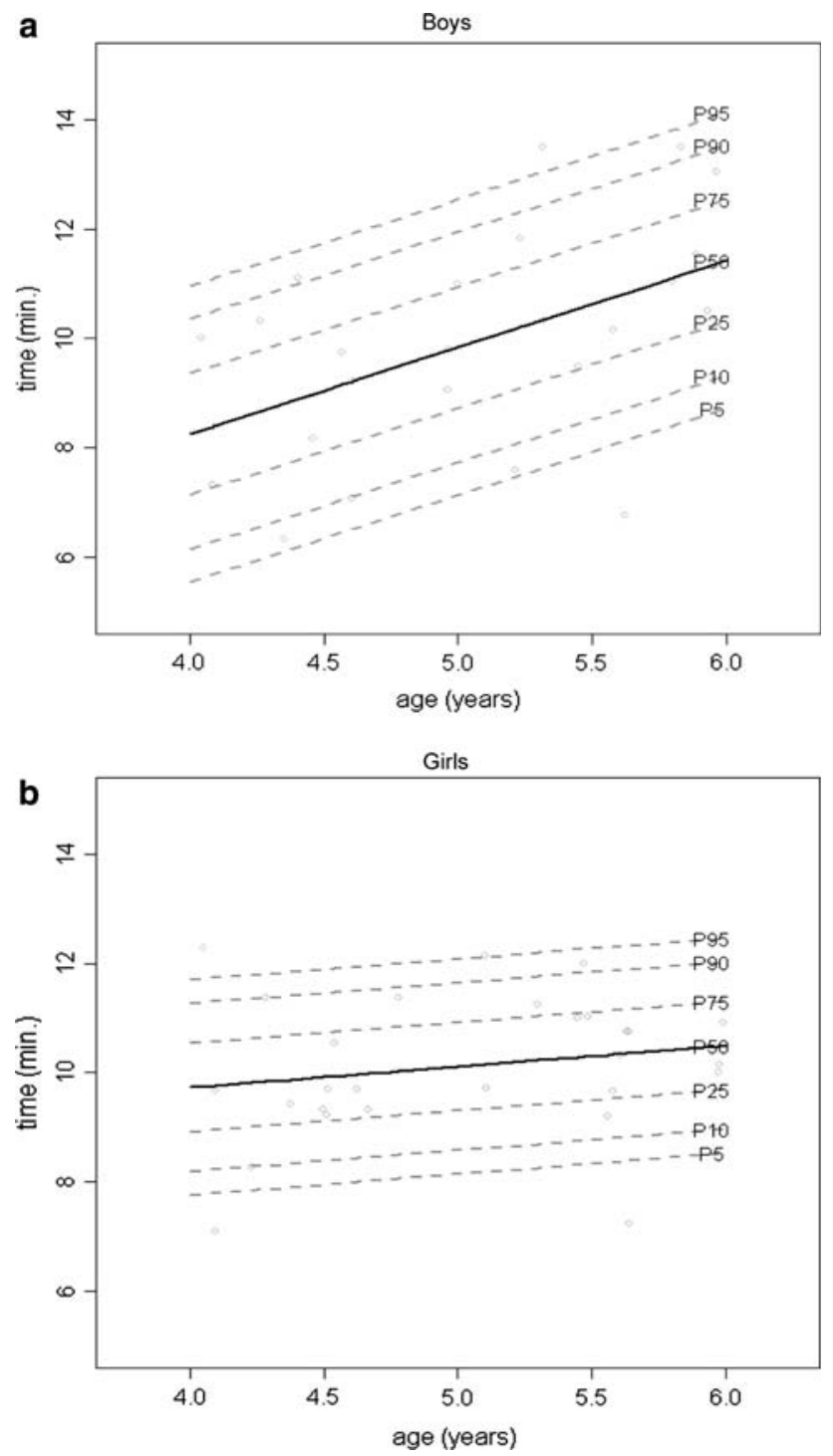

Fig. 2 Centile chart of endurance time in boys (a) and girls (b)

A significant learning effect was observed, as reflected by higher endurance times when children performed a second test. Cumming et al. (1978) showed high test-retest reproducibility for the Bruce protocol in 20 schoolchildren aged 7 to 13 years. We suggest that 4- and 5-year-olds are more sensitive to the habituation with walking on a treadmill. Therefore, we derived our new reference values only from tests performed by children without former experience with the Bruce treadmill protocol.

In our study we did not measure gas exchange parameters. In a clinical setting there is often no metabolic cart available. Moreover, the use of a facemask or mouthpiece might frighten young children. Cumming et al. (1978) reported a strong correlation between the maximal endurance time and maximal oxygen uptake. Endurance time is

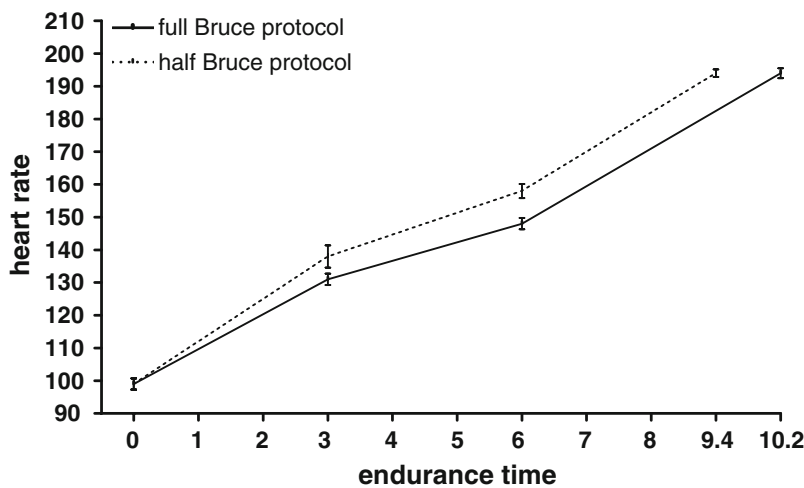

Fig. 3 Increase of heart rate. Continuous line full Bruce protocol; dotted lines half-Bruce protocol. Heart rate in bpm, Endurance time in min. Paired-samples $t$ test: difference in heart rate at 3 min, mean difference $=6, \mathrm{SD}=24, P=0.09$; difference in heart rate at $6 \mathrm{~min}$, mean difference $=10, \mathrm{SD}=14, P \leq 0.01$

therefore a good alternative for testing young children in the clinical setting (Cumming et al. 1978).

\section{Conclusions}

In the current study, we present new reference values for the original Bruce treadmill protocol in healthy children aged 4 and 5 years old. The maximal exercise capacity seems to have deteriorated when we compare our results with those of an earlier study in The Netherlands (Binkhorst et al. 1992). The endurance times on the original and the half-Bruce protocol are not exchangeable. So, we recommend to use the original Bruce protocol in these young children and to give them the opportunity to hold the guardrail so that maximal performance can be achieved successfully.

Acknowledgments We thank the children and their schools for participation. Wouter Vertegaal, physiotherapist, supervised part of the exercise tests. Ko Hagoort provided editorial advice. Zilveren Kruis Achmea financially supported this study.

Open Access This article is distributed under the terms of the Creative Commons Attribution Noncommercial License which permits any noncommercial use, distribution, and reproduction in any medium, provided the original author(s) and source are credited.

\section{References}

Altman DG (1993) Construction of age-related reference centiles using absolute residuals. Stat Med 12:917-924

Bar-Or O (1983) From physiologic principles to clinical applications. In: 1983 NYS-V (ed) Pediatric sports medicine for the practitioner, pp 75-77

Bar-Or O, Rowland T (2004) Pediatric exercise medicine. From Physiologic Principles to Healthcare Application. Human Kinetics, Champaign 
Binkhorst RA, van 't Hof MA, Saris WHM (1992) Maximale inspanning door kinderen; referentiewaarden voor 6-18 jarige meisjes en jongens [Maximal exercise in children; reference values girls and boys, 6-18 year of age]. Brochure Netherlands Heart Foundation, the Netherlands

Bruce RA, Kusumi F, Hosmer D (1973) Maximal oxygen intake and nomographic assessment of functional aerobic impairment in cardiovascular disease. Am Heart J 85:546-562

Chatrath R, Shenoy R, Serratto M, Thoele DG (2002) Physical fitness of urban American children. Pediatr Cardiol 23:608-612

Cumming GR, Everatt D, Hastman L (1978) Bruce treadmill test in children: normal values in a clinic population. Am J Cardiol 41:69-75

Ellestad M (2003) Stress testing. Oxford University Press, Oxford

Fredriks AM, van Buuren S, Burgmeijer RJ, Meulmeester JF, Beuker RJ, Brugman E, Roede MJ, Verloove-Vanhorick SP, Wit JM (2000a) Continuing positive secular growth change in The Netherlands 1955-1997. Pediatr Res 47:316-323

Fredriks AM, van Buuren S, Wit JM, Verloove-Vanhorick SP (2000b) Body index measurements in 1996-7 compared with 1980. Arch Dis Child 82:107-112
Karila C, de Blic J, Waernessyckle S, Benoist MR, Scheinmann P (2001) Cardiopulmonary exercise testing in children: an individualized protocol for workload increase. Chest 120:81-87

Senn S (2002) Cross-over trials in clinical research. Wiley, New York Statistics Netherlands (2001) Standaard beroepen classificatie [Standard classification of occupations] 1992, Edition 2001. Voorburg/ Heerlen, the Netherlands

Statistics Netherlands (2004) Allochtonen in Nederland [People with a foreign background in the Netherlands]. Voorburg/Heerlen, the Netherlands

Takken T (2007) Inspanningstests [Exercise tests]. Elsevier Health Care, Maarssen

Van der Cammen-van Zijp MHM, van den Berg-Emons RJG, Willemsen SP, Stam HJ, Tibboel D, IJsselstijn H (2009) Exercise capacity in Dutch children; new reference values for the Bruce treadmill protocol. Scand J Med Sci Sports, 2009 Apr 20 (early view)

Wessel HU, Strasburger JF, Mitchell BM (2001) New standards for the Bruce treadmill protocol in children and adolescent. Pediatr Exerc Sci 13:392-401 